OPEN ACCESS

Edited by:

Paola Parrella,

Casa Sollievo della Sofferenza

(IRCCS), Italy

Reviewed by:

Jiaxi Yao,

Fudan University, China

Giulia Mazzaschi,

University Hospital of Parma, Italy

Izidor Kern,

University Clinic of Pulmonary and

Allergic Diseases Golnik, Slovenia

*Correspondence:

Giuseppe Bronte

giuseppe.bronte@irst.emr.it

${ }^{+}$These authors have contributed equally to this work

Specialty section:

This article was submitted to Molecular and Cellular Oncology,

a section of the journal

Frontiers in Cell and Developmental

Biology

Received: 07 September 2021 Accepted: 12 November 2021

Published: 30 November 2021

Citation:

Bravaccini S, Bronte G, Petracci E, Puccetti M, D'Arcangelo M, Ravaioli S, Tumedei MM, Maltoni R, Delmonte A, Cappuzzo $F$ and Crinò L (2021) The

Expression of Programmed Death

Ligand 1 and Vimentin in Resected Non-Metastatic Non-Small-Cell Lung

Cancer: Interplay and

Prognostic Effects.

Front. Cell Dev. Biol. 9:772216.

doi: 10.3389/fcell.2021.772216

\section{The Expression of Programmed Death Ligand 1 and Vimentin in Resected Non-Metastatic Non-Small-Cell Lung Cancer: Interplay and Prognostic Effects}

\author{
Sara Bravaccini ${ }^{1 \dagger}$, Giuseppe Bronte ${ }^{2 *}$, Elisabetta Petracci ${ }^{3 \dagger}$, Maurizio Puccetti ${ }^{4}$, \\ Manolo D'Arcangelo ${ }^{5}$, Sara Ravaioli ${ }^{1}$, Maria Maddalena Tumedei ${ }^{1}$, Roberta Maltoni ${ }^{2}$, \\ Angelo Delmonte ${ }^{2}$, Federico Cappuzzo ${ }^{6}$ and Lucio Crinò ${ }^{2}$
}

${ }^{1}$ Biosciences Laboratory, IRCCS Istituto Romagnolo per lo Studio dei Tumori (IRST) "Dino Amadori", Meldola, Italy, ${ }^{2}$ Department of Medical Oncology, IRCCS Istituto Romagnolo per lo Studio dei Tumori (IRST) "Dino Amadori", Meldola, Italy, ${ }^{3}$ Unit of Biostatistics and Clinical Trials, IRCCS Istituto Romagnolo per lo Studio dei Tumori (IRST) "Dino Amadori", Meldola, Italy, ${ }^{4}$ Azienda Unità Sanitaria Locale (AUSL) Imola, Imola, Italy, ${ }^{5}$ AUSL Romagna, Ospedale Santa Maria delle Croci, Ravenna, Italy, ${ }^{6}$ Istituto Nazionale Tumori "Regina Elena", Rome, Italy

Programmed death ligand 1 (PD-L1) is an immune checkpoint with a role in cancer-related immune evasion. It is a target for cancer immunotherapy and its expression is detected for the use of some immune checkpoint inhibitors in advanced non-small cell lung cancer patients (NSCLC). Vimentin is a key component of the epithelial-to-mesenchymal transition phenotype. Its expression has negative prognostic effects in NSCLC. In this study, we retrospectively evaluated PD-L1 and vimentin expression in tumor cells, immune infiltrate and PD-L1 positive immune infiltrate via immunohistochemistry in tissue samples from resected non-metastatic NSCLC patients. We explored the interplay between PD-L1 and vimentin expression through Spearman's correlation test. We performed univariate analysis through the Cox models for demographic and clinico-pathological variables, and also for dichotomized biomarkers, i.e., PD-L1 and vimentin in tumor cells, both with 1 and $50 \%$ cutoffs. We used Kaplan-Meier method to estimate the overall survival, comparing both vimentin and PD-L1 positive patients with all the others. We found a weak positive correlation between PD-L1 and vimentin expressions in tumor cells $(r=0.25$; $p=0.001)$. We also observed a statistically not significant trend towards a shorter overall survival in patients with both PD-L1 and vimentin expression $>1 \%(\mathrm{HR}=1.36$; $95 \% \mathrm{Cl}$ : $0.96-1.93, p=0.087$ ). In conclusion, these findings suggest that interplay between PD-L1 and vimentin may exist in non-metastatic NSCLC patients and the positivity of both markers in tumor tissue is associated with a trend towards a worse prognosis.

Keywords: NSCLC, PD-L1, vimentin, immune infiltrate, non-metastatic (M0) patients

\footnotetext{
Abbreviations: NSCLC, non-small cell lung cancer; IASLC, International Association for the Study of Lung Cancer; OS, overall survival; ICI, immune checkpoint inhibitor; PD-1, programmed death 1; PD-L1, programmed death ligand 1; IHC, immunohistochemistry; EMT, epithelial-to-mesenchymal transition; HR, hazard ratio; CI, confidence interval; KRAS, Ki-ras2 Kirsten rat sarcoma viral oncogene homolog; EGFR, epidermal growth factor receptor; ALK, anaplastic lymphoma kinase; ROS1, c-ros oncogene 1 .
} 


\section{INTRODUCTION}

Lung cancer is still the leading cause of mortality by cancer, despite recent therapeutic advances. In non-small cell lung cancer (NSCLC) 5-year survival rates are limited to $60 \%$ in patients with stage II and $36 \%$ in those with stage IIIA according to the 8th edition staging by the International Association for the Study of Lung Cancer (IASLC) (Goldstraw et al., 2016). The surgery remains the gold standard treatment for patients in early stages (I-II); it can be considered in selected cases with stage IIIA disease. Post-surgical adjuvant platinum-based chemotherapy is used to achieve a modest improvement of around $5 \%$ in 5 -year overall survival (OS) in patients who underwent complete resection for stage II and IIIA (Pignon et al., 2008). It can be considered in those patients who underwent resection for stage IB NSCLC if the primary tumor is greater than $4 \mathrm{~cm}$. For unresectable stage IIIA or IIIB disease the treatment of choice is represented by concurrent chemoradiation therapy or, as an alternative, sequential chemotherapy followed by definitive radiotherapy (Postmus et al., 2017). In both cases, concurrent or sequential therapy, a consolidation with durvalumab, an immune checkpoint inhibitor (ICI), is approved according to the results of PACIFIC trial (Antonia et al., 2017). ICIs are currently being evaluated as neoadjuvant/adjuvant therapy in combination with standard treatments (Mielgo-Rubio et al., 2020).

The interaction between Programmed Death-1 (PD-1) and its ligand, Programmed Death-Ligand 1 (PD-L1), is an immune checkpoint with a relevant role in the regulation of anti-tumor immune response. These molecules are expressed in tumorinfiltrating immune cells and tumor cells, respectively. In some cases, the expression of PD-L1 evaluated through immunohistochemistry (IHC) helped to provide a predictive biomarker for ICI. Specifically, it allowed patients to be selected for upfront pembrolizumab, an anti-PD-1 ICI, among those with metastatic NSCLC which expressed PD-L1 > 50\%, before the approval of chemoimmunotherapy as first-line treatment.

Epithelial-mesenchymal transition (EMT) may be associated with higher aggressiveness in NSCLC.

It is characterized by the reduction of epithelial features and an increase of mesenchymal ones. In this biological condition tumor cells are more invasive and maintain longer survival leading to higher numbers of circulating tumor cells and metastases (Francart et al., 2018). EMT characteristics include a higher expression of vimentin EMT-related transcription factors, and a remodeling of cell-cell contacts (Tsoukalas et al., 2017). The EMT phenotype was related to PD-L1 upregulation by the combined effect of TGF- $\beta 1$ and TNF- $\alpha$. This could be due to the action of TNF- $\alpha$ on NF- $\kappa B$ stimulation, which increases EMT induction by TGF- $\beta 1$. NF- $\kappa B$ inhibition also blocks PD-L1 expression (Asgarova et al., 2018). These results are supported by the fact that TGF- $\beta 1$ and TNF- $\alpha$ induce global DNA demethylation, including the demethylation of the PD-L1 promoter, which causes higher PD-L1 expression.

Moreover, vimentin has been described as a negative prognostic marker in various cancers (Dongre and Weinberg,
TABLE 1 | Patients characteristics $(n=247)$.

\begin{tabular}{|c|c|c|}
\hline & $N$ & $\%$ \\
\hline \multicolumn{3}{|l|}{ Gender } \\
\hline female & 51 & 20.65 \\
\hline male & 196 & 79.35 \\
\hline missing & - & \\
\hline \multicolumn{3}{|l|}{ Age at surgery, yrs } \\
\hline mean $\pm \mathrm{sd}$ & \multirow{2}{*}{\multicolumn{2}{|c|}{$\begin{array}{c}68.20 \pm 7.85 \\
69(36-87)\end{array}$}} \\
\hline Median (min-max) & & \\
\hline missing & - & \\
\hline \multicolumn{3}{|l|}{ Smoking status } \\
\hline Non-smoker & 6 & 15.38 \\
\hline Former smoker & 20 & 51.28 \\
\hline Current smoker & 13 & 33.33 \\
\hline missing & 208 & \\
\hline \multicolumn{3}{|l|}{ Histology } \\
\hline Non squamous & 148 & 59.92 \\
\hline Squamous & 94 & 38.06 \\
\hline Mixed & 5 & 2.02 \\
\hline missing & - & \\
\hline \multicolumn{3}{|l|}{ Grading } \\
\hline G1 & 10 & 4.31 \\
\hline G2 & 87 & 37.50 \\
\hline G3 & 135 & 58.19 \\
\hline missing & 15 & \\
\hline \multicolumn{3}{|c|}{ Disease stage (8th edition) } \\
\hline IA & 22 & 11.70 \\
\hline IB & 45 & 23.94 \\
\hline$\| \mathrm{A}$ & 20 & 10.64 \\
\hline$\| B$ & 50 & 26.60 \\
\hline IIIA & 51 & 27.13 \\
\hline missing & 59 & \\
\hline \multicolumn{3}{|l|}{ Type of surgery } \\
\hline lobectomy & 153 & 61.94 \\
\hline bilobectomy & 10 & 4.05 \\
\hline pneumonectomy & 38 & 15.38 \\
\hline Atypical resection & 44 & 17.81 \\
\hline Other & 2 & 0.81 \\
\hline missing & - & \\
\hline \multicolumn{3}{|c|}{ Neoadjuvant Chemotherapy } \\
\hline no & 20 & 83.33 \\
\hline yes & 4 & 16.67 \\
\hline missing & 223 & \\
\hline \multicolumn{3}{|c|}{ Adjuvant Chemotherapy } \\
\hline no & 19 & 90.48 \\
\hline yes & 2 & 9.52 \\
\hline missing & 226 & \\
\hline \multicolumn{3}{|c|}{ Post-surgery Radiotherapy } \\
\hline no & 21 & 100.00 \\
\hline yes & - & 0.00 \\
\hline missing & 226 & \\
\hline
\end{tabular}

sd: standard deviation; min: minimum; max: maximum.

2019), and also in NSCLC, as extensively documented by numerous studies, then pooled in a meta-analysis (Ye et al., 2016).

In this paper we evaluated the interplay between PD-L1 and vimentin in a wide population of non-metastatic NSCLC patients. We also explored a potential role of immune infiltrate in tumor tissue and analyzed the prognostic impact of these combined markers. 


\section{MATERIALS AND METHODS}

\section{Study Design}

To investigate the association between Vimentin and PD-L1 expression and immune infiltrate and their separate and combined effects in terms of OS, retrospectively retrieved data for patients consecutively treated at Area Vasta Romagna (AVR) since July, 1997, were used. Our hypothesis is that the increase of vimentin expression levels is associated with an increase of PD-L1 expression levels. We also hypothesized that the patients with both Vimentin and PD-L1 positive IHC expression had a worse prognosis in terms of OS. Second aim of the study was to explore the role of immune infiltrate in terms of quantity and percentage of PD-L1 positive immune cells on OS.

\section{Patients}

Eligibility criteria were $\geq 18$ years old, histological diagnosis of non-metastatic NSCLC (Stage I-IIIA defined by using the latest version of staging system). At least one primary tumor specimen had to be available. From the clinical records we extracted information about histology, stage at diagnosis, date of diagnosis, type of radical surgery, adjuvant chemotherapy, adjuvant radiotherapy, date of death or date of last follow-up visit.

Given that the setting of patients analyzed is early stage NSCLC, the assessment of genetic testing was not required.

The Ethics Committee of AVR reviewed and approved the study protocol (NCT03078959). Patients provided written informed consent according to Italian privacy law and following the principles laid down in the Declaration of Helsinki.

\section{Immunohistochemistry}

NSCLC obtained during surgery was fixed in neutral buffered formalin and embedded in paraffin. Four-micron sections were mounted on positive-charged slides (Bio Optica, Milan, Italy). Biomarker evaluations were done according to European Quality Assurance guidelines. PD-L1 and Vimentin Immunostaining expression was performed using PD-L1 SP263 and Confirm anti-Vimentin V9 (Ventana Medical Systems) antibody clones by Ventana BenchmarkXT staining system (Ventana Medical Systems, Tucson, AZ, United States) with the Optiview DAB Detection Kit (Ventana Medical Systems).

For their detection, tissue sections were incubated for $16 \mathrm{~min}$ with prediluted antibodies by the supplier. Sections were incubated for $16 \mathrm{~min}$ and automatically counterstained with hematoxylin II (Ventana Medical Systems). Placenta was used as positive control for both the biomarkers. Membranous biomarker positivity was detected and semiquantitatively quantified as the percentage ratio between immunopositive tumor cells and the total number of tumor cells.

We defined immune infiltrate considering mainly the tumor infiltrating lymphocytes (TILs). PD-L1 positivity was detected both on tumor cells and on the immune infiltrate. We considered the amount (\%) of the whole immune infiltrate on the total of the cells present in the sample. The PD-L1 positivity on immune infiltrate was evaluated in terms of percentage of $\mathrm{PD}$-L1 positive immune cells on the total number of immune cells without subtyping the TILs identified by using only morphological criteria, due to the low number of sections available to perform further IHC detections.

Vimentin expression has been evaluated only on tumor cells given that its expression in the cells of interstitial area is known to be ubiquitous.

All samples were evaluated by 2 independent observers and any disagreement ( $>10 \%$ of positive cells for the different markers) was resolved by consensus after joint review using a multihead microscope.

\section{Statistical Analysis}

Data were summarized using mean \pm standard deviation (sd), median and minimum and maximum values, for continuous variables. Categorical variables were reported as natural frequency and percentage. Correlation among variables was measured through the Spearman correlation coefficient.

Given the heavily asymmetric distribution of the considered biomarkers, characterized by a "spike at zero," the association between demographic and clinical covariates as well as with the OS was assessed using dichotomized variables on the $1 \%$ value. Patients with expression values lower than $1 \%$ were considered "negative" for the biomarkers whereas those with values equal or greater than $1 \%$, were considered as "positive." Additional exploratory analyses considering a 50\% cutoff ("negative" less than $50 \%$, "positive" if equal or greater than $50 \%$ ) as well as considering the biomarkers as continuous variables were also performed. These cutoffs were chosen because these are commonly used in clinical practice for PD-L1 characterization for the use of pembrolizumab in first- or second-line treatment for advanced NSCLC. The Chi-square test or the Fisher Exact test was used for the association between the biomarker as dichotomous variable and categorical covariates whereas the Wilcoxon-Mann-Whitney or the Kruskal Wallis test was used for the association between the biomarker as continuous variable and categorical covariates.

OS was defined as the time from surgery until death for any cause or last patient visit by April, 2016. The Kaplan-Meier method was used to estimate the OS function whereas the Log-rank test was used for survival curves comparison. Hazard ratios (HRs) and corresponsing 95\% confidence intervals (CIs) were obtained applying the Cox proportional hazards model. To investigate the association between the biomarkers as continuous variables, the method proposed by Royston and Lorenz was also applied (Royston et al., 2010; Lorenz et al., 2017; Lorenz et al., 2019). The proportional hazards assumption was tested using Schoenfeld residuals. For all the analyses a two-sided $p$-value $<0.05$ was considered statistically significant. The analyses were carried with STATA 15.0 (College Station, Texas, United States).

\section{RESULTS}

In this retrospective study we included 247 patients who met the eligibility criteria. The majority of patients were male (79\%), former or current smokers $(85 \%)$, with a non-squamous histology $(60 \%)$. The mean age at surgery was $68.20 \pm 7.85$ years. The stages were variably distributed: $36 \%$ stage I, $37 \%$ stage II, $27 \%$ stage IIIA. As 
TABLE 2 | Descriptive statistics of IHC biomarkers.

\begin{tabular}{|c|c|c|c|c|c|c|c|}
\hline & missing & Mean & sd & Median & $\min$ & Max & IQR \\
\hline PD-L1+ tumor cells & - & 20.76 & 33.24 & 0 & 0 & 100 & 30 \\
\hline Vim + tumor cells & - & 10.96 & 23.19 & 0 & 0 & 100 & 5 \\
\hline Immune infiltrate & 12 & 16.01 & 14.99 & 10 & 1 & 90 & 15 \\
\hline PD-L1+ immune infiltrate & 12 & 5.53 & 9.36 & 2 & 0 & 80 & 10 \\
\hline
\end{tabular}

IHC: immunohistochemistry; PD-L1: programmed death ligand 1; Vim: vimentin; sd: standard deviation; min: minimum; max: maximum; IQR: interquartile range.

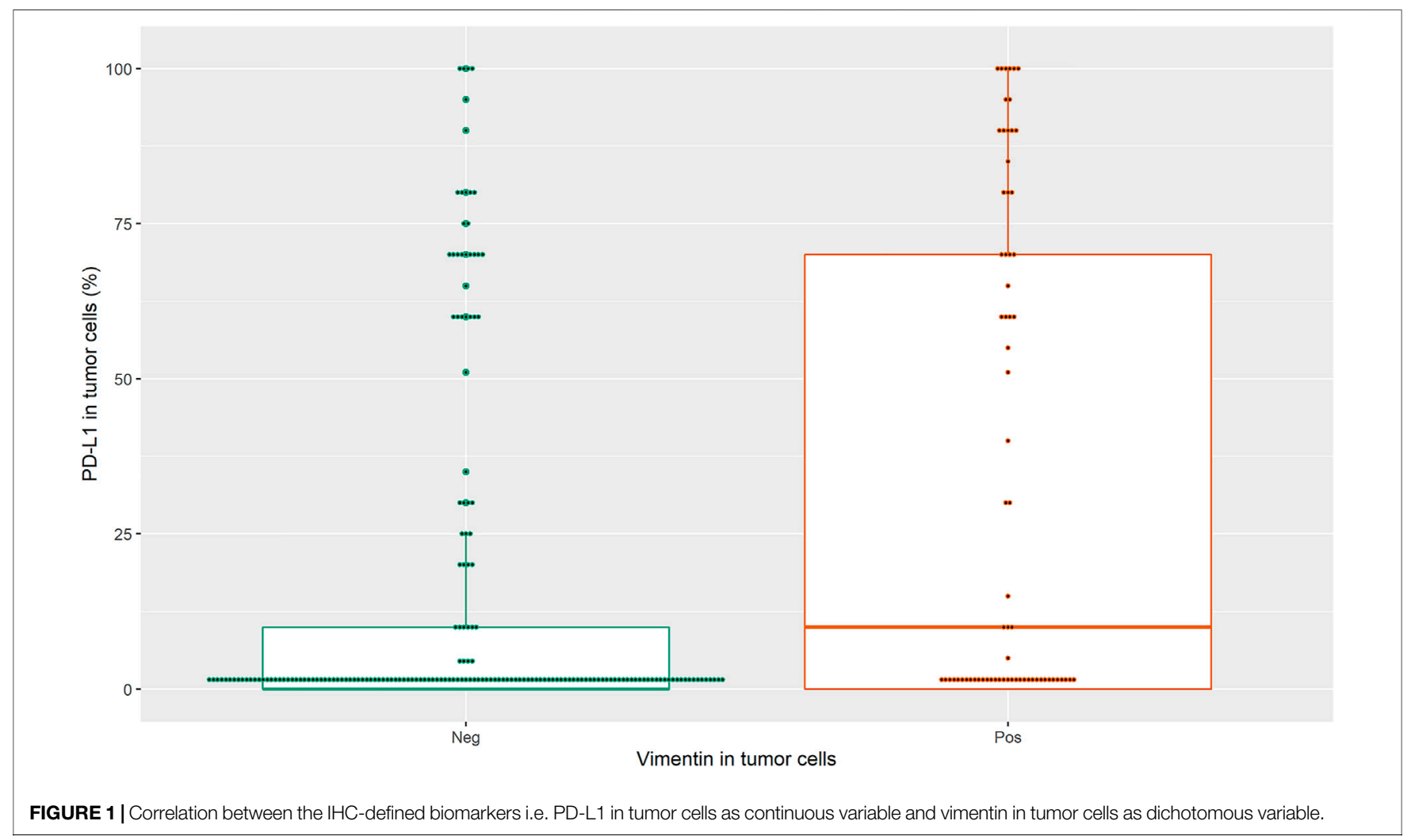

regards surgery, the majority of patients (62\%) underwent lobectomy. As regards cancer treatments, $17 \%$ received neoadjuvant chemotherapy, $10 \%$ received adjuvant chemotherapy and none of the patients was treated with post-surgical radiation therapy (Table 1). The mean values of PD-L1 expression in tumor cells, vimentin expression in tumor cells, immune infiltrate, and PD-L1-positive immune infiltrate with standard deviations are reported in Table 2, together with medians and minimum and maximum values. PD-L1 median percentage in tumor cells was equal to zero as well as the vimentin median percentage in tumor cells. The median values for the immune infiltrate and the PD-L1-positive immune infiltrate were equal to 10 and 2, respectively.

We measured the correlation between the IHC-defined biomarkers as continuous variables through the Spearman correlation coefficient. We found a weak positive correlation between PD-L1 and vimentin expressions in tumor cells $(r=$ $0.25 ; p=0.001)$. The comparison of PD-L1 expression in tumor cells between high and low vimentin expression (1\% of cutoff value) by using Wilcoxon-Mann-Whitney resulted in a statistically significant difference $(p$-value $<0.001)$, Figure 1.

A similar result was obtained using the $50 \%$ cutoff for vimentin expression (data not shown).

The tumor cells were negative for PD-L1 or vimentin with both the cutoff values in the majority of patients. Tumor cells were PD-L1-negative in $76 \%$ of cases with $50 \%$ cutoff and $57 \%$ with $1 \%$ cutoff. The same cells were vimentin-negative in $89 \%$ of cases with $50 \%$ cutoff and $72 \%$ with $1 \%$ cutoff. For the samples from 12 patients the information about immune infiltrate was missing. However, almost all cases (97\%) were negative for immune infiltrate with $50 \%$ cutoff value, but all the samples had an immune infiltrate $\geq 1 \%$. In $99 \%$ of cases the immune infiltrate had a PD-L1 expression $<50 \%$, but with $1 \%$ cutoff value PD-L1-positive immune infiltrate was present in the majority of patients (67\%) (Table 3).

No significant associations were found between the biomarkers and demographic and clinico-pathological covariates (results not shown). 
TABLE 3 | Distribution of biomarkers' values with different cutoffs.

\begin{tabular}{|c|c|c|c|c|}
\hline & \multicolumn{2}{|c|}{$50 \%$ cutoff } & \multicolumn{2}{|c|}{$1 \%$ cutoff } \\
\hline & $N$ & $\%$ & $n$ & $\%$ \\
\hline \multicolumn{5}{|c|}{ PD-L1 in tumor cells } \\
\hline Negative & 188 & 76.11 & 140 & 56.68 \\
\hline Positive & 59 & 23.89 & 107 & 43.32 \\
\hline missing & - & & - & \\
\hline \multicolumn{5}{|c|}{ Vimentin in tumor cells } \\
\hline Negative & 220 & 89.07 & 207 & 71.63 \\
\hline Positive & 27 & 10.93 & 82 & 28.37 \\
\hline missing & - & & - & \\
\hline \multicolumn{5}{|c|}{ Immune infiltrate } \\
\hline Negative & 227 & 96.60 & 0 & 0.00 \\
\hline Positive & 8 & 3.40 & 235 & 100.00 \\
\hline missing & 12 & & 12 & \\
\hline \multicolumn{5}{|c|}{ PD-L1+ immune infiltrate } \\
\hline Negative & 233 & 99.15 & 78 & 33.19 \\
\hline Positive & 2 & 0.85 & 157 & 66.81 \\
\hline missing & 12 & & 12 & \\
\hline
\end{tabular}

TABLE 4 | Results from univariate Cox models for demographic and clinicopathological variables.

\begin{tabular}{|c|c|c|}
\hline & HR (95\% Cl) & $\mathbf{P}$ \\
\hline \multicolumn{3}{|l|}{ Gender } \\
\hline Female & 1 (ref) & \\
\hline Male & $1.22(0.88-1.70)$ & 0.239 \\
\hline \multicolumn{3}{|l|}{ Age } \\
\hline$<70$ & 1 (ref) & \\
\hline$\geq 70$ & $1.40(1.15-1.95)$ & 0.003 \\
\hline \multicolumn{3}{|l|}{ Smoking status } \\
\hline non smoker & 1 (ref) & \\
\hline ex smoker & $0.64(0.23-1.78)$ & 0.399 \\
\hline Smoker & $0.77(0.26-2.26)$ & 0.632 \\
\hline \multicolumn{3}{|l|}{ Histology } \\
\hline non squamous & 1 (ref) & \\
\hline Squamous & $1.03(0.78-1.35)$ & 0.848 \\
\hline Mixed & $0.98(0.36-2.65)$ & 0.964 \\
\hline \multicolumn{3}{|l|}{ Grading } \\
\hline G1 & 1 (ref) & \\
\hline G2 & $1.71(0.83-3.55)$ & 0.148 \\
\hline G3 & $2.24(1.09-4.59)$ & 0.027 \\
\hline \multicolumn{3}{|l|}{ Disease stage } \\
\hline 1 & 1 (ref) & \\
\hline ॥ & $1.40(0.97-2.01)$ & 0.072 \\
\hline III & 2.15 (1.52-3.32) & $<0.001$ \\
\hline
\end{tabular}

HR: hazard ratio; Cl: confidence interval; $P$ : $\mathrm{p}$-value; ref: reference.

The bold values refer to statistically significant values $(\mathrm{p}<0.05)$.

We also performed an univariate analysis for OS through the Cox models as regards the available demographic and clinicopathological variables, including gender, age, smoking status, histology, grading and disease stage, and type of surgery. We found statistically significant differences for age ( $<70$ vs. $\geq$ 70 years), grading (G1 vs. G3) and disease stage (I vs. III) (Table 4).

An univariate analysis for OS was also performed for the dichotomized biomarkers (Table 5). Note that for the immune infiltrate only eight patients had a value $\geq 50 \%$ whereas for the PD-L1+immune infiltrate only two patients presented with a value $\geq 50 \%$. However, we did not find statistically significant
TABLE 5 | Results from univariate Cox models for dichotomized biomarkers.

\begin{tabular}{|c|c|c|c|c|}
\hline & \multicolumn{2}{|c|}{$50 \%$ cutoff } & \multicolumn{2}{|c|}{$1 \%$ cutoff } \\
\hline & HR $(95 \% \mathrm{Cl})$ & $\mathbf{P}$ & HR $(95 \% \mathrm{Cl})$ & $\mathbf{P}$ \\
\hline \multicolumn{5}{|c|}{ PD-L1 in tumor cells } \\
\hline Negative & 1 (ref) & & 1 (ref) & \\
\hline Positive & $0.95(0.69-1.30)$ & 0.732 & $1.01(0.78-1.30)$ & 0.961 \\
\hline \multicolumn{5}{|c|}{ Vimentin in tumor cells } \\
\hline Negative & 1 (ref) & & 1 (ref) & \\
\hline Positive & $1.14(0.72-1.81)$ & 0.565 & $1.19(0.90-1.57)$ & 0.219 \\
\hline \multicolumn{5}{|c|}{ Immune infiltrate } \\
\hline Negative & 1 (ref) & & 1 (ref) & \\
\hline Positive & $0.66(0.29-1.48)$ & 0.312 & $-^{\mathrm{a}}$ & \\
\hline \multicolumn{5}{|c|}{ PD-L1+ immune infiltrate } \\
\hline Negative & 1 (ref) & & 1 (ref) & \\
\hline Positive & $1.33(0.33-5.37)$ & 0.687 & $1.19(0.89-1.58)$ & 0.243 \\
\hline
\end{tabular}

HR: hazard ratio; Cl: confidence interval; $P$ : $\mathrm{p}$-value; ref: reference.

${ }^{a} \mathrm{HRs}$, and $95 \% \mathrm{Cls}$, could not be estimated because no patients presented with a value $<1 \%$.

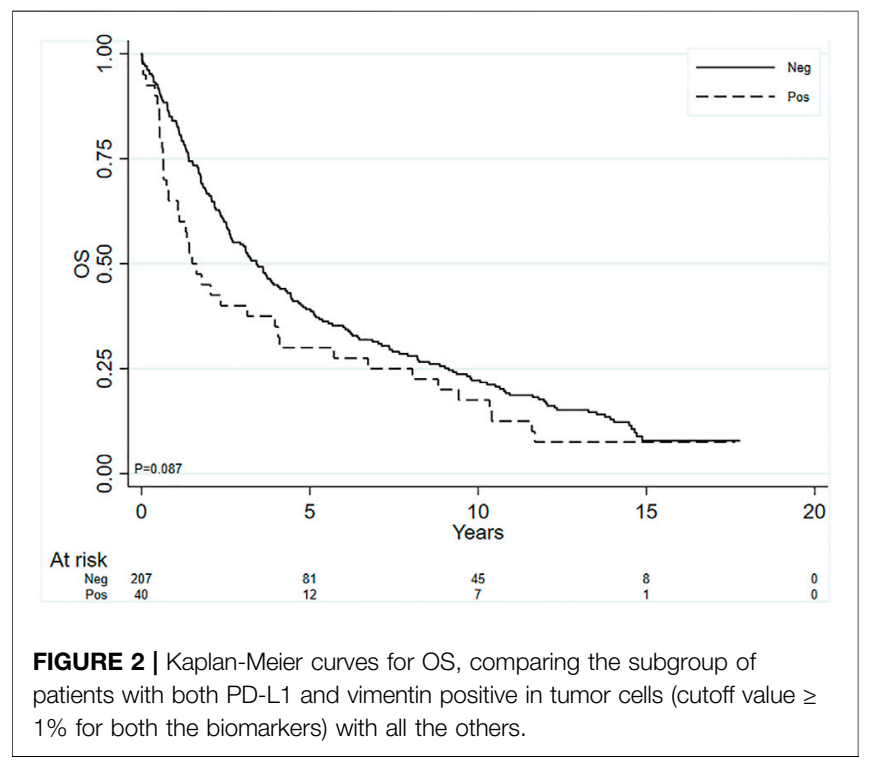

differences for the analyzed biomarkers (Table 5). No statistically significant associations for all four biomarkers were observed also when considered as continuous variables (results not shown).

Finally, given that we hypothesized that the patients with both Vimentin and PD-L1 positive (40 patients) could have a worse prognosis, we compared this subgroup of patients with all other patients $(n=207)$. We found a trend even if not statistically significant toward a worse survival in this double positive subgroup using $1 \%$ cutoff value for both the biomarkers (HR = 1.36; 95\% CI: $0.96-1.93, p=0.087)$. Figure 2 shows the KaplanMeier curves for the two groups. Such a trend in significance disappeared when age and stage were also included in the Cox model. When considering the $50 \%$ cutoff no association was observed $(\mathrm{HR}=1.03$; 95\% CI: $0.60-1.77, p=0.912)$, Figure 3 .

Figures 4, 5 show the expression of PD-L1 and vimentin in some tissue slides to highlight the differential expression of these biomarkers in tumor cells and infiltrate with immune cells. 


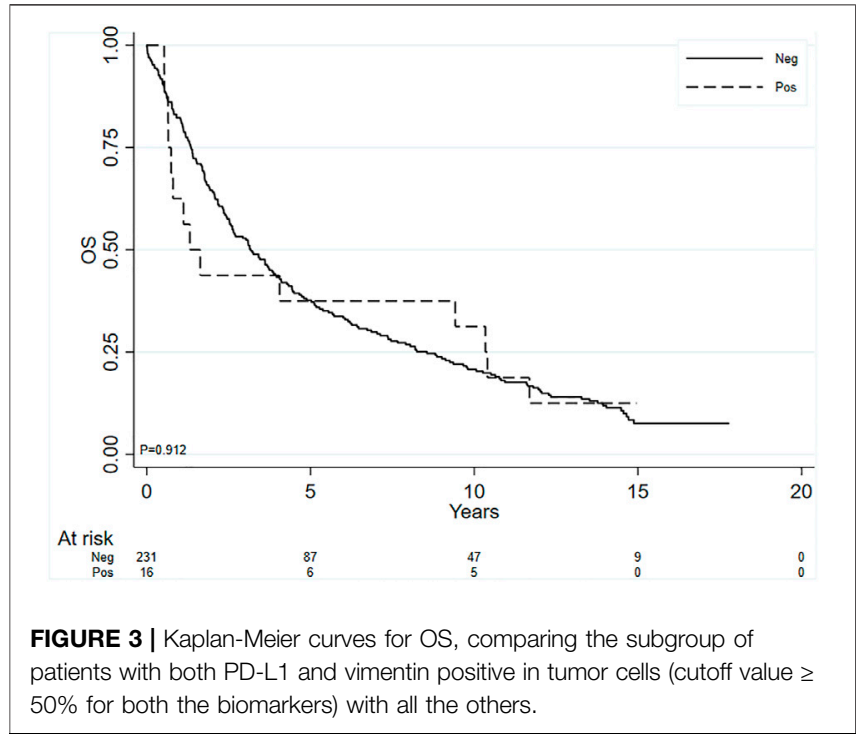

\section{DISCUSSION}

In the last few years PD-L1 expression in tumor tissue increased its importance because of the availability of ICIs which target the PD-1/PD-L1 pathway. However, these drugs have not yet reached a role in resected non-metastatic NSCLC. Vimentin expression is not directly involved in the treatment of this neoplasm, but it has a key role in the EMT process, which seems to be involved in resistance to various cancer treatments. Its prognostic role has been widely studied in NSCLC patients. Few studies explored its association with PD-L1 expression (Asgarova et al., 2018).

We previously retrospectively analyzed data on patients with advanced NSCLC consecutively enrolled in a clinical study in our center. PD-L1 and vimentin expression were detected by immunohistochemistry (Bronte et al., 2021). We used $\geq 1 \%$ and $\geq 50 \%$ as cutoff values to define PD-L1 positivity on immune cells because these cutoff values are the same used on tumor cells in the clinical practice to select patients for immunotherapy. A weak positive association between PD-L1 and vimentin in advanced NSCLC suggests a potential interplay between these biomarkers. Moreover, given our previous results (Bronte et al., 2021), the established prognostic value of these biomarkers in other subset of disease, we investigated their expression in an earlier disease setting, non-metastatic NSCLC patients. We also explored a potential role of immune infiltrate in tumor tissue and analyzed the prognostic impact of these combined markers.

We found a weak positive correlation between PD-L1 and vimentin expressions in tumor cells as well as a correlation between the quantity of immune cells and \% of immune cells PD-L1 positive. In particular, we retrospectively analyzed Vimentin and PD-L1 expression in tumor cells and immune infiltrate to study their separate and combined effects in terms of OS.

In our study a positive association between the percentage of PD-L1 positive tumor cells and the percentage of vimentin in tumor cells was seen.

The interplay between EMT markers and immune checkpoint inhibitors (Chouaib et al., 2014; Chen et al., 2015; De Matteis et al., 2019) has been previously reported. The concurrent expression of PD-L1 and EMT phenotype was described both in tumor tissue and circulating tumor cells (Kim et al., 2016; Raimondi et al., 2017; Manjunath et al., 2019). The effect of EMT on immune evasion is exerted through the regulation of PD-L1 expression (Asgarova et al., 2018) as a consequence of a synergistic exposure to TGF- $\beta 1$ and TNF- $\alpha$. These factors determine global DNA demethylation, also in the promoter of
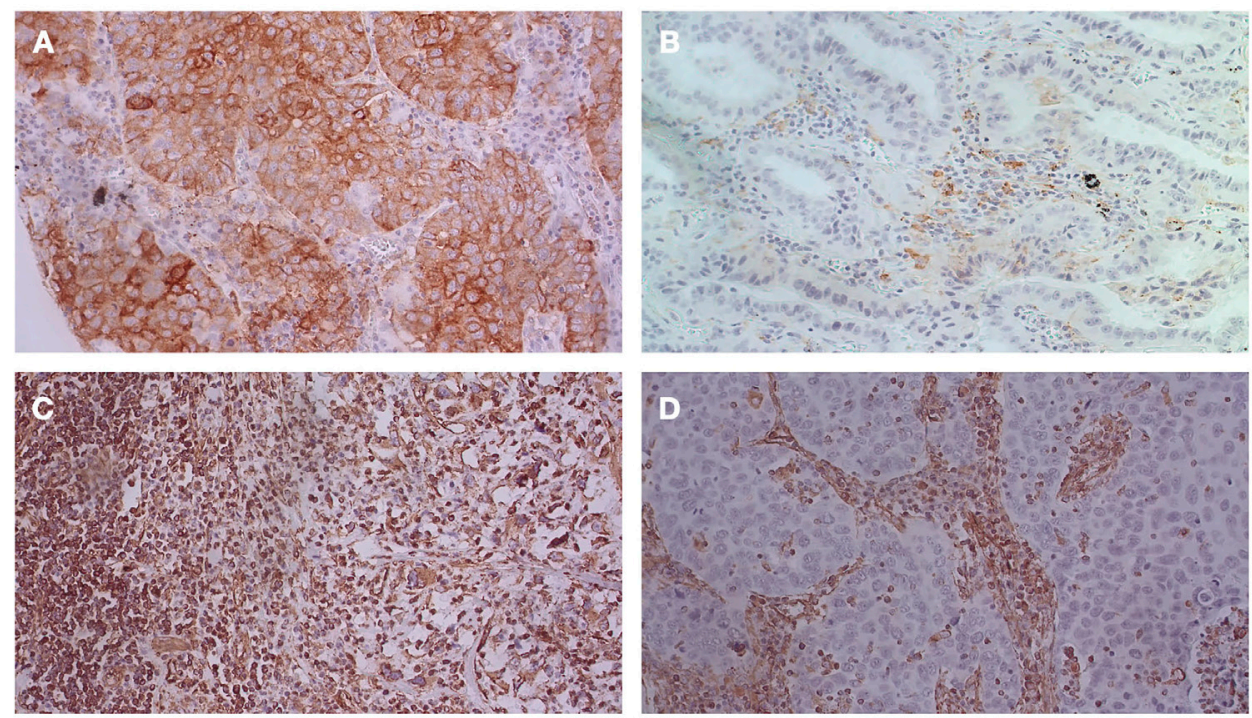

FIGURE 4 | IHC analysis for PD-L1 and vimentin: (A) PD-L1 positive in both tumor cells and infiltrate with immune cells (40X magnification); (B) PD-L1 positive in infiltrate with immune cells, but negative in tumor cells (40X magnification); (C) Vimentin expression observed both in tumor cells and immune cells (20X magnification); (D) Vimentin expression observed only in immune cells (20X magnification). 


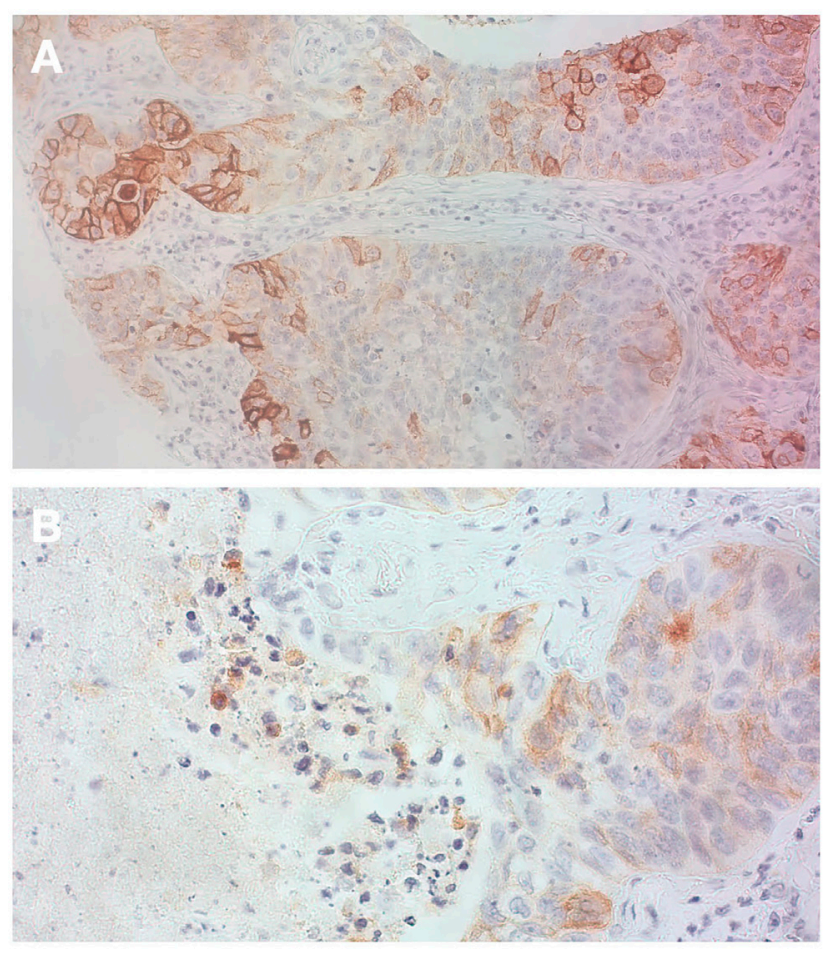

FIGURE 5 | IHC analysis for PD-L1 (A) This picture highlights heterogeneity in term of PD-L1 expression in tumor cells (20 X magnification); (B) Weak PD-L1 positivity mainly on tumor cells (also macrophages and very few stromal lymphocytes are positive) (40 X magnification).

the gene encoding for PD-L1, and consequently a higher PD-L1 expression is achieved.

Ancel and colleagues reported that a cut-off $\geq 25 \%$ vimentinpositive tumor cells was significantly associated with poor tumor differentiation even if it was not sufficient to predict a worse prognosis. However, these authors reported that concurrent high PD-L1 and vimentin expressions in early-stage NSCLC patients were more clearly associated with a shorter OS (Ancel et al., 2019). Such results are concordant with our finding of a trend even if it was not statistically significant of a worse survival in the patients harboring double positivity for PD-L1 and Vimentin using $1 \%$ cutoff value for both the biomarkers.

Our study has some limitations, because it is retrospective and this population of non-metastatic NSCLC patients is heterogeneous,

\section{REFERENCES}

Ancel, J., Birembaut, P., Dewolf, M., Durlach, A., Nawrocki-Raby, B., Dalstein, V., et al. (2019). Programmed Death-Ligand 1 and Vimentin: A Tandem Marker as Prognostic Factor in NSCLC. Cancers (Basel) 11 (10). doi:10.3390/cancers11101411

Antonia, S. J., Villegas, A., Daniel, D., Vicente, D., Murakami, S., Hui, R., et al. (2017). Durvalumab after Chemoradiotherapy in Stage III Non-small-cell Lung Cancer. N. Engl. J. Med. 377 (20), 1919-1929. doi:10.1056/nejmoal709937

Asgarova, A., Asgarov, K., Godet, Y., Peixoto, P., Nadaradjane, A., Boyer-Guittaut, M., et al. (2018). PD-L1 Expression Is Regulated by Both DNA Methylation and mainly in terms of disease stage, type of surgery and subsequent treatments. Another strong limitation is the use of archival tissue for IHC analysis and the limited number of patients included in this study. A prospective study could allow the analysis in fresh tissue to evaluate more biomarkers associated with EMT and immune evasion. Moreover, proper cut-off values to define vimentin and PD-L1 positivity have to be established. Probably, we did not find any prognostic significance of immune infiltrate because the overall amount of lymphocytes is not sufficient to determine a prognostic effect, but perhaps the specific subsets of lymphocytes (e.g. $\mathrm{CD}^{+}, \mathrm{CD}^{+}$, etc.) could impact on prognosis, but we could not determine these subsets because of the few slides available. Anyway, our findings suggest that EMT markers and immune escape markers could be intended as components of the same process.

\section{DATA AVAILABILITY STATEMENT}

The raw data supporting the conclusion of this article will be made available by the authors, without undue reservation.

\section{ETHICS STATEMENT}

The studies involving human participants were reviewed and approved by the Ethics Committee of AVR. The patients/ participants provided their written informed consent to participate in this study.

\section{AUTHOR CONTRIBUTIONS}

GB, FC, and LC conceived experiments; SB, RM, and EP conceived experiments and analysed data; SR, MMT, and MP carried out experiments; $\mathrm{AD}$ and MD'A collected data. All authors contributed to manuscript revision, read, and approved the submitted version.

\section{ACKNOWLEDGMENTS}

The authors thank Grainne Tierney and Cristiano Verna for editorial assistance.

NF-kB during EMT Signaling in Non-small Cell Lung Carcinoma. Oncoimmunology 7 (5), e1423170. doi:10.1080/2162402x.2017.1423170

Bronte, G., Puccetti, M., Petracci, E., Landi, L., Cravero, P., Scodes, S., et al. (2021). The Interplay between Programmed Death Ligand 1 and Vimentin in Advanced Nonsmall-cell Lung Cancer. Front. Oncol. 11, 669839. doi:10.3389/fonc.2021.669839

Chen, L., Heymach, J. V., Qin, F. X.-F., and Gibbons, D. L. (2015). The Mutually Regulatory Loop of Epithelial-Mesenchymal Transition and Immunosuppression in Cancer Progression. Oncoimmunology 4 (5), e1002731. doi:10.1080/ 2162402x.2014.1002731

Chouaib, S., Janji, B., Tittarelli, A., Eggermont, A., and Thiery, J. P. (2014). Tumor Plasticity Interferes with Anti-tumor Immunity. Crit. Rev. Immunol. 34 (2), 91-102. doi:10.1615/critrevimmunol.2014010183 
De Matteis, S., Canale, M., Verlicchi, A., Bronte, G., Delmonte, A., Crinò, L., et al. (2019). Advances in Molecular Mechanisms and Immunotherapy Involving the Immune Cell-Promoted Epithelial-To-Mesenchymal Transition in Lung Cancer. J. Oncol. 2019, 7475364. doi:10.1155/2019/7475364

Dongre, A., and Weinberg, R. A. (2019). New Insights into the Mechanisms of Epithelial-Mesenchymal Transition and Implications for Cancer. Nat. Rev. Mol. Cel Biol. 20 (2), 69-84. doi:10.1038/s41580-018-0080-4

Francart, M.-E., Lambert, J., Vanwynsberghe, A. M., Thompson, E. W., Bourcy, M., Polette, M., et al. (2018). Epithelial-mesenchymal Plasticity and Circulating Tumor Cells: Travel Companions to Metastases. Dev. Dyn. 247 (3), 432-450. doi:10.1002/dvdy.24506

Goldstraw, P., Chansky, K., Crowley, J., Rami-Porta, R., Asamura, H., Eberhardt, W. E., et al. (2016). The IASLC Lung Cancer Staging Project: Proposals for Revision of the TNM Stage Groupings in the Forthcoming (Eighth) Edition of the TNM Classification for Lung Cancer. J. Thorac. Oncol. 11 (1), 39-51. doi:10.1016/j.jtho.2015.09.009

Kim, S., Koh, J., Kim, M.-Y., Kwon, D., Go, H., Kim, Y. A., et al. (2016). PD-L1 Expression Is Associated with Epithelial-To-Mesenchymal Transition in Adenocarcinoma of the Lung. Hum. Pathol. 58, 7-14. doi:10.1016/j.humpath.2016.07.007

Lorenz, E., Jenkner, C., Sauerbrei, W., and Becher, H. (2019). Modeling Exposures with a Spike at Zero: Simulation Study and Practical Application to Survival Data. Biostatistics Epidemiol. 3 (1), 23-37. doi:10.1080/24709360.2019.1580463

Lorenz, E., Jenkner, C., Sauerbrei, W., and Becher, H. (2017). Modeling Variables with a Spike at Zero: Examples and Practical Recommendations. Am. J. Epidemiol. 185 (8), 650-660. doi:10.1093/aje/kww122

Manjunath, Y., Upparahalli, S. V., Avella, D. M., Deroche, C. B., Kimchi, E. T., Staveley-O'Carroll, K. F., et al. (2019). PD-L1 Expression with Epithelial Mesenchymal Transition of Circulating Tumor Cells Is Associated with Poor Survival in Curatively Resected Non-small Cell Lung Cancer. Cancers 11 (6), 806. doi:10.3390/cancers 11060806

Mielgo-Rubio, X., Calvo, V., Luna, J., Remon, J., Martín, M., Berraondo, P., et al. (2020). Immunotherapy Moves to the Early-Stage Setting in Non-small Cell Lung Cancer: Emerging Evidence and the Role of Biomarkers. Cancers 12 (11), 3459. doi:10.3390/cancers12113459

Pignon, J.-P., Tribodet, H., Scagliotti, G. V., Douillard, J.-Y., Shepherd, F. A., Stephens, R. J., et al.LACE Collaborative Group (2008). Lung Adjuvant Cisplatin Evaluation: a Pooled Analysis by the LACE Collaborative Group. Jco 26 (21), 3552-3559. doi:10.1200/jco.2007.13.9030

Postmus, P. E., Kerr, K. M., Oudkerk, M., Senan, S., Waller, D. A., Vansteenkiste, J., et al.ESMO Guidelines Committee (2017). Early and Locally Advanced Non- small-cell Lung Cancer (NSCLC): ESMO Clinical Practice Guidelines for Diagnosis, Treatment and Follow-Up. Ann. Oncol. 28 (Suppl. 1_4), iv1-iv21. doi:10.1093/annonc/mdx222

Raimondi, C., Carpino, G., Nicolazzo, C., Gradilone, A., Gianni, W., Gelibter, A., et al. (2017). PD-L1 and Epithelial-Mesenchymal Transition in Circulating Tumor Cells from Non-small Cell Lung Cancer Patients: A Molecular Shield to Evade Immune System?. Oncoimmunology 6 (12), e1315488. doi:10.1080/ 2162402x.2017.1315488

Royston, P., Sauerbrei, W., and Becher, H. (2010). Modelling Continuous Exposures with a 'spike' at Zero: a New Procedure Based on Fractional Polynomials. Statist. Med. 29 (11), 1219-1227. doi:10.1002/sim.3864

Tsoukalas, N., Aravantinou-Fatorou, E., Tolia, M., Giaginis, C., Galanopoulos, M., Kiakou, M., et al. (2017). Epithelial-Mesenchymal Transition in Non Small-Cell Lung Cancer. Anticancer Res. 37 (4), 1773-1778. doi:10.21873/ anticanres. 11510

Ye, Z., Zhang, X., Luo, Y., Li, S., Huang, L., Li, Z., et al. (2016). Prognostic Values of Vimentin Expression and its Clinicopathological Significance in Non-small Cell Lung Cancer: A Meta-Analysis of Observational Studies with 4118 Cases. PLoS One 11 (9), e0163162. doi:10.1371/ journal.pone. 0163162

Conflict of Interest: The authors declare that the research was conducted in the absence of any commercial or financial relationships that could be construed as a potential conflict of interest.

Publisher's Note: All claims expressed in this article are solely those of the authors and do not necessarily represent those of their affiliated organizations, or those of the publisher, the editors and the reviewers. Any product that may be evaluated in this article, or claim that may be made by its manufacturer, is not guaranteed or endorsed by the publisher.

Copyright (C) 2021 Bravaccini, Bronte, Petracci, Puccetti, D’Arcangelo, Ravaioli, Tumedei, Maltoni, Delmonte, Cappuzzo and Crino. This is an open-access article distributed under the terms of the Creative Commons Attribution License (CC BY). The use, distribution or reproduction in other forums is permitted, provided the original author(s) and the copyright owner(s) are credited and that the original publication in this journal is cited, in accordance with accepted academic practice. No use, distribution or reproduction is permitted which does not comply with these terms. 